\title{
Other Cells Interferences of Power Controlled CDMA Systems
}

\author{
Samad S. Kolahi \\ Unitec Institute of Technology \\ Private Bag 92025, Auckland, NEW ZEALAND \\ Phone: +6498154321 \\ e-mail: skolahi@unitec.ac.nz
}

\begin{abstract}
In this paper, using a simulation and modelling technique, the other cells interferences factor is obtained and compared with previous upper bounds. The effect of propagation parameters on other cells interferences is investigated. Two scenarios are studied, when the mobile chooses the nearest base station and when it chooses the least attenuation base station. The results indicate that that the other cells interference factor can be substantially less than the upper bounds obtained in previous works.
\end{abstract}

Key terms: CDMA, other-cells interferences

\section{INRODUCTION}

Mobile communications have enjoyed rapid growth during the last several years. As the amount of bandwidth available remains the same, capacity planning becomes an important issue in cellular systems. CDMA system capacity decreases with the amount of interference. In CDMA all calls interfere with one another as they all use the same frequency range. Each base station not only receives interference from mobiles in the home cell (intra-cell interference) but also from mobiles located in neighbouring cells (inter-cell interference). An in-depth knowledge of parameters affecting the interference is therefore required. In [1] an upper bound for other cells interference factor $f$ (the ratio of other cells interferences to within cell interferences) was presented. Such a parameter is commonly used in approximating other cells interferences [2]. In this paper, a simulation and modelling method is used to determine the other cells interferences factor and the results are compared with the upper bounds in [1]. In addition, the effect of propagation parameters on other cells interferences is investigated.

The organization of this paper is as follows. In the next section the system model is discussed. The simulation results are then presented. The last section presents conclusions.

\section{SYSTEM MODEL}

A cellular CDMA network is considered (a home cell and tiers of neighbouring cells) with a base station located at the centre of each cell. The tiers of neighbouring cells are added until any further addition has very little effect. All cells are assumed to be homogeneous in every respect. Users are uniformly distributed over the cell area. The reverse link (from mobile to cell site) is modelled as it is the limiting link due to its inferior performance compared to forward link [3]. The calls to the CDMA system are modelled as Poisson $[2,4]$ with mean arrival rate of $\lambda$ calls/second and mean call holding time of $1 / \mu$ seconds per call. In queuing terms, this is a $\mathrm{M} / \mathrm{M} / \infty$ system which is being used for CDMA systems modelling [2]. Once the mobile call has been admitted, it stays in the system during its call holding time which is modelled as negative exponential with probability density function:

$$
f(t)=\mu e^{-\mu t}
$$

Poisson arrivals mean the inter-arrivals have a negative exponential probability distribution function. If the arrival rate is $\lambda$ calls/sec, the inter-arrival times have mean of $1 / \lambda$ with probability distribution function:

$$
f(t)=\lambda e^{-\lambda t}
$$

Handoff requests arrivals also follow a Poisson distribution $[5,6]$. The sum of two Poisson process (with $\lambda_{1}$ and $\lambda_{2}$ ) is another Poisson process with $\lambda=\lambda_{1}+\lambda_{2}$ [7]. The total arrival rate $\lambda$ is defined as the sum of the normal call arrival rate $\lambda_{1}$ and handoff traffic $\lambda_{2}$.

$$
f(t)=\left(\lambda_{1}+\lambda_{2}\right) e^{-\left(\lambda_{1}+\lambda_{1}\right) t}
$$

Traffic density (offered traffic load), $\lambda / \mu$, represents the excess of the arrival rate versus departure rate. $\lambda / \mu$ is measured in Erlang.

All calls are allowed into the system (soft capacity) if they meet the required Quality of Service (QoS). Any calls not meeting this required quality are not permitted to enter the system but are blocked. The required call quality depends on the amount of interference received, that in turn depends on the power of signals received from interfering calls.

The power of signals received is the product of the transmitted power, $m$ th power of the distance and a lognormal shadowing parameter $(\xi)$ with mean zero and standard deviation of $\sigma \xi$. This shadowing parameter varies with different terrains. Assuming $S_{\mathrm{t}}$ and $S$ are the transmitted and received power respectively, we have: 


$$
S=S_{t} r^{-m} 10^{\xi / 10}
$$

The interference from the $j$ th mobile in neighbouring cell $i$ is expressed as [8]:

$$
\begin{aligned}
& (I)_{i j}=S \frac{r_{m}^{m}}{10^{\xi_{m} / 10}} \cdot \frac{10^{\xi_{0} / 10}}{r_{0}^{m}} \\
& (I / S)_{i j}=\left(r_{m} / r_{0}\right)^{m} 10^{\xi_{0}-\xi_{m} / 10}
\end{aligned}
$$

where $S$ is the received signal strength at home base station, $r_{m}$ is the random distance to corresponding home cell base station (figure 1), $r_{0}$ is the distance to the neighbouring cell, $\xi_{0}$ and $\xi_{\mathrm{m}}$ are lognormal (Gaussian in $\mathrm{dB}$ ) random variable with zero mean and standard deviation $\sigma_{\xi}$ representing shadowing parameter in neighbouring and home cell, and $m$ is path loss exponent.

Total other cell interference $I_{\mathrm{o}}$ is interference produced by all users who are power controlled by other base stations. Assuming a CDMA system with $M$ outer cells and $N$ users per cell, the total other user interferences-to-signal ratio $(I / S)_{o}$ is:

$$
(I / S)_{o}=\sum_{i=1}^{M} \sum_{j=1}^{N} I_{i j} / S
$$

On each arrival of a new call, the total interference is determined. This involves repeatedly generating $r_{m}$ between 0 and 1 and uniform random variable $\theta$ between 0 and $2 \pi$. Using figure $1, \mathrm{r}_{0}$ can be calculated for each user as:

$$
r_{0}=\sqrt{r_{m}^{2}+d^{2}+2 d r_{m} \operatorname{Cos} \theta}
$$

Using the above equations, the total received power from interfering cells at the home base station is calculated by considering the path loss exponent $m$ and shadowing parameter $\xi_{0}-\xi_{\mathrm{m}}$. If independent lognormal variables $\xi_{\mathrm{m}}$ and $\xi_{0}$ have average zero and variance of $\sigma_{\xi}^{2}, \xi_{0}-\xi_{\mathrm{m}}$ has mean zero and variance $2 \sigma \xi_{\xi}^{2}$. For each interfering call, a lognormal shadowing parameter, $\xi_{0}-\xi_{\mathrm{m}}$, is generated with mean zero and total standard deviation $\sigma_{\mathrm{T}}=\sigma_{\xi} \sqrt{2}$.

The transmission quality of a CDMA call may then be calculated in terms of the energy per bit over interference spectral density $E_{b} / N_{0}[8]$.

$$
\begin{aligned}
& \frac{E_{b}}{N_{\mathrm{o}}}=\frac{S / R}{I / W}=\frac{S / R}{\left((N-1) S+I_{o}+\eta\right) / W}= \\
& \frac{W / R}{(N-1)+(I / S)_{o}+\eta / S}
\end{aligned}
$$

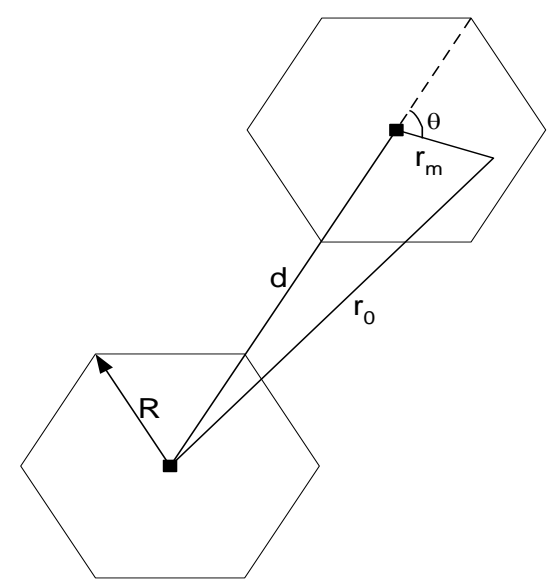

Figure 1. Interfering call distance to home cell

$(I / S)_{0}$ is the ratio of other cells interference to the received signal strength $(S)$ at home base station, $\eta$ is background noise, $W / R$ is Processing Gain, $W$ is available spread bandwidth, and $R$ is data rate.

Taking voice activity into consideration:

$$
\frac{E_{b}}{N_{0}}=\frac{W / R}{\alpha(N-1)+\alpha(I / S)_{o}+\eta / S}
$$

On each call arrival $E_{b} / N_{0}$ is determined $(7,10)$ to decide if the call is accepted to the system or is blocked. If the required call quality of $\mathrm{E}_{\mathrm{b}} / \mathrm{N}_{0} \geq 5.012(7 \mathrm{~dB})$, or $B E R<10^{-3}$, is not achieved, the call is blocked. Other parameter values were $W / R=125$, $\alpha=0.375$ (voice activity parameter), $\lambda / \mu=21$ Erlang, $S / \eta=-1 \mathrm{~dB}$ (the received signal power to background noise), and $m=4$.

\section{SIMULATION RESULTS}

The simulation is performed for one million arrivals and on each arrival, the other cells interferences factor is determined using equations $(6,7,11)$. These values are then averaged over the simulation period. Note that the value of $S$ (power received at base station assuming perfect power control) is not required when calculating the ratio.

$$
f=\frac{\alpha I_{o}}{\alpha(N-1) S}=\frac{(I / S)_{o}}{(N-1)}
$$

The simulation results are obtained for two scenarios: (a) when the home cell is the closest cell; (b) when the home cell is the cell that provides the least attenuation [8]:

$$
\left(r_{m} / r_{0}\right)^{m} 10^{\xi_{0}-\xi_{m} / 10} \leq 1
$$

Tables 1 and 2 show relative other cells interference factors. $\mathrm{Nc}=1$ signifies that the mobile chooses the closest cell while the $\mathrm{Nc}=2$ signifies that the mobile chooses the least attenuation of two base stations as the home cell. The results are compared to the upper bound analytical results given in [1]. This paper's results appear to be smoother than in [1] where changing total shadowing from 10 to 12 has changed $f$ from 6.23 to 20 while our simulation model indicated change of 2.28 to 4.14 . 


\begin{tabular}{|c|c|c|c|c|}
\hline & \multicolumn{2}{|c|}{$\mathrm{Nc}=1$} & \multicolumn{2}{c|}{$\mathrm{Nc}=2$} \\
\hline$\sigma \mathrm{\sigma}(\mathrm{dB})$ & $f$ & Bound [1] & $f$ & Bound [1] \\
\hline 2 & 0.50 & 0.49 & 0.44 & 0.43 \\
\hline 4 & 0.60 & 0.67 & 0.46 & 0.47 \\
\hline 6 & 0.84 & 1.14 & 0.48 & 0.56 \\
\hline 8 & 1.32 & 2.40 & 0.52 & 0.77 \\
\hline 10 & 2.28 & 6.23 & 0.57 & 1.28 \\
\hline 12 & 4.14 & 20.00 & 0.60 & 2.62 \\
\hline
\end{tabular}

Table 1: Relative other cell interference factor $(f)$ for $\mathrm{m}=4$

\begin{tabular}{|c|c|c|c|c|}
\hline \multicolumn{3}{|c|}{$\mathrm{Nc}=1$} & $\mathrm{Nc}=2$ \\
\hline$m$ & $f$ & Bound[1] & $f$ & Bound[1] \\
\hline 3.0 & 2.54 & - & 1.05 & 1.60 \\
\hline 3.5 & 1.76 & - & 0.72 & - \\
\hline 4.0 & 1.32 & 2.40 & 0.52 & 0.77 \\
\hline 4.5 & 1.05 & - & 0.40 & - \\
\hline 5.0 & 0.88 & - & 0.32 & 0.47 \\
\hline
\end{tabular}

Table 2: Relative other cell interference factor $(f)$ for $\sigma_{\mathrm{T}}=8 \mathrm{~dB}$.

The results confirm the results in [1] where by choosing the least attenuation of two links, a significant gain can be achieved. In table 1 , for differential shadowing parameter of $8 \mathrm{~dB}$, the other cells interference will reduce from 1.32 to 0.52 if the link with least attenuation is used as home cell. Note that using the upper bound results of [1] will result in lower system capacity than actual capacity due to higher other cells interference factor. The above table provides an other cells interferences factor $(f)$ that could be used to provide a better understanding of capacity issues in CDMA systems. This factor was found to be independent of traffic load.

\section{CONCLUSIONS}

In this paper, using a simulation and modelling method, the other cells interferences factors are determined. The results were compared with the upper bounds obtained in [1]. It is concluded that the upper bounds could be much higher that the actual other cells interferences factors. As other cells interferences factor is commonly used in analytical calculations, the upper bounds could result in lower system capacity than the actual capacity.

\section{REFERENCES}

[1] A. J. Viterbi and A. M. Viterbi, "Other-Cell Interference in Cellular Power-Controlled CDMA," IEEE Transaction on Communications, 42 (2), pp. 1501- 1504, 1994.

[2] A.M. Viterbi and A. J. Viterbi, "Erlang Capacity of a Power Controlled CDMA System," IEEE Journal on Selected Areas in Communications, vol 11(6), pp. 892-899, 1993.
[3] J.S. Evans and D. Everitt, "Analysis of Reverse Link Traffic Capacity for Cellular Mobile Communication Networks Employing Code Division Multiple Access," Australian Telecommunication Networks and Applications Conference, Melbourne, pp. 775-780, 1994.

[4] J.S. Evans and D. Everitt, "On the Teletraffic Capacity of CDMA Cellular Networks," IEEE Transaction on Vehicular Technology, vol 48(1), pp. 153-165, 1999.

[5] R.P. Narrainen and F. Takawira, "A Queueing Model for CDMA Cellular Networks Using Soft Capacity" IEEE Africon, Vol 1, pp. 147 -152, 1999.

[6] D. M. Lee, "Performance Simulation of Call Capacity for Base Station in CDMA System", IEEE $6^{\text {th }}$ International Conference on Universal Personal Communications, Vol 2, pp. 603-607, 1997.

[7] P. T. Strait, "Probability and Statistics with Applications", chapter 8, second edition, Harcourt Brace Jovanovich Publishers, New York, 1983.

[8] K.S. Gilhousen, I.M. Jacobs, R. Padovani, A. J. Viterbi , et al, "On the Capacity of a Cellular CDMA System," IEEE Transaction on Vehicular Technology, vol 40(2), pp. 303-311, 1991. 